bestand die Prüfung, wie der Cod. med. Hamb. sie vorschreibt. Möchte diese Arbeit, welche nur zwei bekannte Melodien in Eine verschmolzen und die Tonarten etwas beleuchtet hat, sich eines gleichen Schicksals erfreuen *).

Dritte Abtheilung.

\title{
Toxilkologi e.
}

\section{Ueber Arsenikvergiftungen; \\ von \\ Orfila.}

Sitzungen in der Facultät der Medicin zu Paris.

Herr $\mathrm{O}_{\text {r }}$ fil a hatte sich vorgenommen in vier Sitzungen zu beweisen:

1) Dafs die Arsenichtsänre und der Brechweinstein, in den Nahrungskanal oder auf das subcutane Zellgewebe gebracht, absorbirt, dem Blute beigemischt und zu allen Organen der thierischen Oekonomie übergeführt werden.

2) Dafs diese Gifte eine gewisse Zeit in den Eingeweiden und Muskeln verweilen, wo ihre Gegenwart nachgewiesen werden kann; dals aber von den ersten Stunden der Vergiftung an, ein Theil der absorbirten Portion diese Gewebe verlälst und durch den Harn weggeführt wird.

3) Dafs diese Ausscheidung, die für den Brechweinstein rascher vor sich geht als für die Arsenichtsäure, mehre Tage fortdauert, bis die Gewebe des Giftes gänzlich entleert sind.

4) Dals es daher vortheilhaft und selbst unerläfslich ist, bei der Behandlung der Vergiftung durch diese Gifte, die Urinsecretion zu begünstigen.

5) Dafs es in den meisten Fällen möglich ist, zu unterscheiden, ob Arsenichtsäure oder Brechweinstein,

*) Nordisches Centralblatt für die Pharmacie. 1840. V, 457. 
welche man aus den Eingeweiden einer Leiche erhalten hat, die Vergiftung bewirkten.

6) Dal's die geeignetsten Methoden zur Auffindung kleiner Portionen des absorbirten Giftes darin bestehen, die organischen Materien gänzlich oder zum gröfsesten Theil zu zerstören, durch Verkohlen mittelst concentr. Salpetersäure oder Zersetzen mittelst: Salpeter, und Behandeln der Producte in dem modificirten Marsh'schen Apparate.

7) Dafs es leicht ist, Arsen und Antimon, unter Form von Flecken, zn unterscheiden, und sich zu überzeugen, dafs diese Hecken weder ron dem Apparate noch von den in Anwendung gresetzten Reagentien herriihren.

8) Dals in den Knochen der Menschen und melirer Thiere cine in Wasser unlüsliche arsenikalische Verbindung rorhanden ist.

9) Dars man aus dem Muskelfleische iles Menschen eine Substanz ausziehen kann, die er zusammengesetzt glaubt aus einer sehr licinen Proportion Arsenik, Schwefel und einer organischen Snbstanz.

10) Dafs man in dem 'Terrain gewisser Kirchhöfe aufserordentlich kleine Spuren von Arsenik findet, welche von liochendem Wasser nicht gelöst werden.

11) Dafs man bei gerichtlichen Fällen die Irrthiimer leicht vermeiden kann, welche auf den ersten Anblick durch die Gegrenwart von Arsenil in den Knochen, Muskeln und dem Terrain verschiedener Kirchhöfe entstehen können.

\section{Erste Sitzung, den 25. October 1810.}

Die Sitzung wurde um $10 \frac{3}{5}$ Uhr eröfnet. Hr. Orfila setzt den Plan, den er bei seinen Vorlesungen zu befolgen beabsichtigrt, auscinander. Er legt den Oesophagus eines lileinen weifsen auf dem Kopfe schwarzgefleckten Hundes blofs, unterbindet den Kanal und zeigt an, dafs die Ligratur bis zum folgenden 'Tage 2 Uhr unterhalten bleiben, der Ilund aber bis zur Sitzung an 25. November autbewahrt werlen sulle, $n$ m zu beweisen, dars er darch die Operation nicht merklich belästignt worden sei. 
In den Magren eines Hundes von mittler Grösse wurden 60 Centigrm. Arsenichtsiunre, in $50 \mathrm{Grm}$. Vasser gelöst, eingebracht; die nöthigen Ligaturen wurden angebracht.

Auf das subcutane Zellgewebe eines andern Hundes wurden 15 Centigrm. Arsenichtsäure, feingepulvert, applicirt, und die Ränder der Wunde durch einige Nadeln vereinigt.

Ein anderer Hund wurde wie der vorige mit 15 Centigr. Brechweinstein vergiftet.

Ein Hund wurde, ohne vergiftet zu sein, anfgehangen. Man öfnete ihn sogleich und nahm die Leber, die Milz, die Nieren, das Herz und die Lungen aus, welche getrocknet wurden, das trockne Product wurde durch sein dreifaches Gewicht reiner Salpetersäure von $31^{\circ}$ Areomet. in einer reinen Porcellanschale verkohlt, die erhaltene Kohle zwanzig Minuten lang mit kochendem dest. Wasser behandelt, die filtrirte röthlich-braune Flïssigkeit in einem zuvor geprüften Marsh'schen Apparat behandelt; sie gab keine Spur eines arsenikalischen oder andern Fleckens, selbst nach Verlauf einer halben Stunde.

Um $10 \frac{1}{2}$ Uhr fing man an in ciner grofsen neuen Porcellanschale sechs Kilogrm. Muskelfleisch von einem Menschen mit dest. Wasser und einer Unze reinem Kali zu liochen.

Auch die in Kleine Stücken zerschnittene Leber eines erwachsenen Menschen wurde mit VVasser in einer Porcellanschale gekocht.

Die Iinochen eines Menschen wurden bei einer hohen und einer niedrigen Temperalur calcinirt, hierauf gepulvert und gesiebt; die ersten gaben ein weifses, die andern ein schwarzes Palces. 9 Unz. eines jeden dieser Pulver warden in zwei Porcellanschalen durch 4 Unz. reine Sehwefelsäure und Wasser zersetzt. Die Mischungen wurden einige Tage lang sich selbst iiberlassen.

Da um $1 \frac{3}{4}$ Uhr der vergiftete Ifund noch nicht todt war, so wurde er anfgehangen, und gleich nach seinem Tode zog man aus der Blase olngefähr $24 \mathrm{Grm}$. 
Urin; man sonderte die Leber, ohne Verletzung des Verdauungskanals aus. Der vierte Theil der Leber wurde getrocknet und durch Salpetersäure verkohlt, die Eingeweide des normalen Hundes, und darauf die Fohle 20 Minuten lang mit Wasser gekocht. Die filtrirte röthliche Flüssigkeit war kaum in den zuvor geprüften Marsh'schen Apparat gebracht, als sie zahlreiche grofse braune und glänzende Arsenikflecken gab. Der Urin dieses Thieres gab gelbe glänzende arsenikalische Flecken, als er in einer gewissen Menge in einem zuvor geprüften Marsh'schen Apparate behandelt wurde.

Der Urin des gehenkten normalen Hundes gab keine Spur von Arsenik.

Marsh'scher Apparat. Man liefs Wasser, Zink und rectificirte Schwefelsäure in ein Glas geben, und ohngefïhr eine Stunde lang Wasserstoffas entwickeln, die Flamme mochte zwei Linien lang sein; man erhielt nicht die geringste Spur Arsenik auf der Porcellanfläche.

Fin anderer Apparat, in welchem ebenfalls Wasser, Zink und Schwefelsänre sich befand und der nach $10 \mathrm{Mi}$ nuten langer Thätiglieit keine Spur von Arsenik gegeben hatte, gab sogleich zahlreiche und grofse braune Arseniliflecken, als man in denselben einen Tropfen reiner concentrirter Auflüsung von Arsenichtsäure gebracht hatte.

In ein mitSchwefelwasserstoffgas gefüll tes Glas brachte man ein Gemenge von zwei Theilen Wasser und einem Theil Schwefelsäure; es setzte sich nichts ab als ein nilchweifser Schwefel, während dasselbe Gemenge, als man noch cinen Tropfen einer Auflösung von Arsenichtsänre zugesetzt hatie, einen Niederschlag von hellgelbem Schwefelarsenik gab.

Charaktere der Arsenik- und Antimonialflecken. Das Anseln der Arsenikflecken ist von dem der Antimonialflecken verschieden. Werden die ersten der Einwirkung der Flamme des reinen Wasserstoffgases ausgesetzt, so verschwinden sie fast in demselben Augenblick; die andern dehnen sich aus, werden weniger intensiv, und 
sind noch nach einigen Minuten der Einwirkung vorhanden. Beide verschwinden auf der Stelle durch Einwirkung reiner concentr. Salpetersäure, und läfst man die Flüssigkeiten verdunsten, so erhält man von den Arsenikftecken einen gelblich - weifsen und mit den Antimonialflecken einen gelben Rückstand. Beim Erkalten der Schälchen nnd wenn man auf beide Rückstände zwei Tropfen einer nicht sauren Auflösung von salpetersaurem Silber bringt, giebt der Arsenikrückstand ziegelrothes arsenich tsaures Silber, während der Antimonialrückstand nicht verändert wird.

Eine Unze trocknes Gelatin wurde mit eben so viel reiner Salpetersäure von $41^{\circ}$ vermischt, schon nach $6 \mathrm{Mi}$ nuten hatte man eine sehr schöne Kohle.

Die Herren Husson und Pelletier, Mitglieder der Commission, assistirten bei den von $10_{\frac{1}{4}}^{1}$ bis $4 \frac{1}{2} \mathrm{Uhr}$, wo die Sitzung aufgehoben wurde, angestellten Versuchen. Die verschiedenen Producte der Operationen, die während der Sitzung nicht beendet warden, legte man unter Siegel und vertraute das Pettschaft Hrn, H usson an.

Zweite Sitzung, am 26. October.

1) Um 9 Uhr Morgens und in Gegenwart der Herren Husson, Amusat, Caventou u. s. w. wurden, nachdem man sich zuvor von der Integrität der Siegel überzeugt hatte, die verschiedenen Schalen, in welchen die Rüclistände der vorigen Operationén sich befanden, aus dem Kabinet genommen.

2) Das Decoct des Muskelfleisches, erhalten nach fünfstündigem Kochen, wurde durch Leinwand gegeben, zur Trockne verdunstet, durch reine concentrirte Salpetersäure verkohlt, die Kohle zwanzig Minuten mit heifsem dest. Wasser in Berührung gelassen, filtrirt, und die Flüssiglieit, gehörig bezeichnet, hingestellt.

3) Das Decoct der nicht. vergifteten Leber eines Erwachsenen wurde ebenfalls colirt und verdunstet, wachdem es mit $60 \mathrm{Grm}$. reinem Salpeter vermischt worden.

Das getrocknete Product wurde in einem hessischen 
Tiegel bei Rothglühhitze verbrannt, der Rückstand mit Wasser behandelt und durch Schwefelsäure zersetzit, und die Flüssigkeit nach einstündigem Kochen für den weitern Versuch anfbewahrt.

4) Man lief's die drei Viertel der Leber des früher durch 60 Centirrm. Arsenichtsäure vergifteten Thieres mit dest. Wasser drei Stunden lang in einer Porcellanschale kochen, das Decoct durch Leinwand coliren, mit $60 \mathrm{Grm}$. reinen Salpeter mischen, zur Trockne abrauchen und den Rückstand in einem neuen hessischen Tiegel verbrennen. Der Rückstand wurde mit Wasser und Schwefelsäure wie zuvor behandelt und dann für die weitern Versuche zurückgestellt.

5) Um $9 \frac{1}{2}$ Uhr vergiftete man einen Hund mittler Grörse, indem man in seinen Magen 60 Centigrm. Brechweinstein, aufgelöst in $150 \mathrm{Grm}$. Wasser, brachte. Die nothwendigen Ligaturen wurden gemacht; da um $\mathbf{l}_{\frac{3}{4}}^{3} \mathrm{Uhr}$ das Thier noch nicht todt war, wurde es gehenkt. Die Blase enthielt nur ohngefähr $6 \mathrm{Grm}$. Urin, der gesammelt wurde. Die Hälfte der Leber dieses Hundes wurde getrocknet und verkohl, die Kohle eine Viertelstunde lang mit Chlorwasserstoffsäure, die mit $\frac{1}{4}$ ihres Gewichts Wasser vermischt war, behandelt, und die filtrirte Flïssioglieit aufbewahrt. Der Harn des Thieres $(6 \mathrm{Grm}$.) wurde zur Trockne verdunstet, der Rückstand verkohlt, mit Chlorwasserstoffsäure, die mit $\frac{1}{4}$ Wasser verdünnt war, gekocht und das Filtrat aufbewahrt.

6) Auf dieselbe Weise wurden $150 \mathrm{Grm}$. Harn von dem gestern mit 15 Centigrm. auf dem Schenkel applicirten Brechweinstein vergifteten Hunde, behandelt. Da dieser Hund hente um $10 \mathrm{Uhr}$ noch nicht todt war, so wurde er gehenkt.

7) Um 10 Uhr Morgens hatte MT... 560 Grm. krystallisirten Salpeter und eben so viel Salpeter in Masse in einem Marmormörser gemengt. Die Hälfte dieses Gemenges (500 Grm.) wurden durch $980 \mathrm{Grm}$. reiner concentrirter Scliwefelsäure in einer Porcellanschale, mittelst Erwärmen, zersetzt. Die mit $\mathbf{V}$ asser verdünnte 
Flüssigkeit wurde filtrirt und zur Seite gestellt. Die andere Hälfte des Salpeters wurde auf dieselbe Weise behandelt, nachdem man zuvor noch 1 Centigrm. Arseniksäure zugesetzt hatte.

Um 2 Uhr las Hr. Orfila das Protokoll der vorigen Sitzung, welches nach Rechtbefund von den Mitgliedern der Commission unterzeichnet wurde.

Orfila liês die Ligatur, die gestern um den Oesophagus des weifsen auf dem Kopfe schwarzgefleckten Hundes gelegt worden war, wegnehmen; das Thier war ziemlich munter, als es hereingelbracht wurde, während des Aufsuchens der durch das Aufschwellen des Fleisches und der schon entzündeten Wundränder ziemlich tief liegenden Ligatur wurde es ohnmächtig.

Es wurden jetzt die beiden in No. 7. erhaltenen Flüssigkeiten untersucht; beide wurden in zwei grofse zuvor geprüfte Marsh'sche Apparate gebracht. Die Gasentwicklung war so stark, namentlich mit der arsenikalischen Flüssigkeit, dafs man die Hälfte herausnehmen und eine beträchtliche Menge Wasser zusetzen mufste. Das Gas wurde angezündet, und obgleich die Flamme stark war, so setzten sich doch sogrleich auf der Porcellanfläche zahlreiche und grofse Arsenikflecken ab. Die andere Flüssigkeit, welcher keine Arsenilsäure zugemischt worden war, war weniger sauer als die vorhergehende, weil sie durch das Kocheu eine ziemliche Menge Schwefelsäure verloren hatte, so dafs man kaum noch den dritten Theil der Flüssigkeit erhielt, und das Uebrige durch Wasser ersetzte. Das Gas brannte mit eiver 2-3 Linien langen Flamme, die aber weit schwächer war als die aus der vorigen Flüssigkeit. Es war unmöglich, jetzt irgend eine Spur eines Arsenikfleckens oder eines Fleckens irgend anderer Art zu erhalten.

Die Flüssigkeit No. 2, die vom Muskelfleisch kam, in einem zuvor geprüften Marsh'schen Apparate untersucht, ab nur einigge grofse weifse opake Flecken, und eine kleine Zahl anderer brauner nicht glänzender.

Die Flïissigkeit No. 3. von der normalen Leber gab 
in einem zuvor geprüften Marsh'schen Apparate keine Spur eines Fleckens.

Die Flïssigkeit No. 4, welche von $\frac{3}{4}$ der Leber des Tages zuvor mit 60 Centigrm. Arsenichtsäure vergifteten Hundes erhalten war, wurde in einen zuvor geprüften Marsh'schen A pparat gebracht; sie gab eine grofse Zahl brauner glänzender Flecken.

Die $6 \mathrm{Grm}$. Harn von dem am Morgen vergifteten Hunde gaben kein Arsenik zu erkennen (No. 5.).

Die Hälfte der Leber dieses Thieres hatte eine Flüssigkeit gegeben (S. No.5.), die in den zuvor geprüften Marsh'schen Apparat gebracht, fast sogleich zahlreiche Antimonflecken gab.

Die Flüssigkeit von dem Harn des mit $15 \mathrm{Grm}$. auf dem Schenkel applicirten Brechweinstein vergifteten Hundes (No. 6.) gab, in dem zuvor geprüften Marsh'schen Apparate, sofort eine aufserordentliche Menge von Antimonialflecken.

Der gestern mit 15 Centigrm. auf dem Schenkel applicirter Arsenichtsäure vergiftete Hund war während der Nacht gestorben. Die Blase war leer, obgleich das Thier keinen Harn gelassen hatte. Man wusch dieses Organ mit dest. Wasser aus und brachte diese Flüssigkeit mit ohngefähr 2 Unz. Olivenöl in einen Marsh'schen Apparat; nach einigen Augenblicken erhielt man drei bis vier kleine gelbe glänzende augenscheinlich arsenikalische Flecken.

Die Sitzung wurde um $3 \frac{3}{1}$ Uhr anfgehoben, und die beiden Schalen mit den calcinirten Knochen aus der Sitzung vom 25. unter Siegel gestellt.

Orfila setzte das nothwendige Verfahren auseinander :

1) Um Arsenik von Antimon zu unterscheiden, wenn ein Individuum durch Brechweinstein vergiftet wurde.

2) Um zu entdecken, ob in dem Falle, wo ein Individuum durch Arsenichtsäure vergiftet ist, und mit Eisenperoxyd behandelt wurde, die Arsenikflecken von 
den als Gegengift angewandten Peroxyde herrühren oder nicht.

3) Um za entscheiden, ob in den Falle, wo das Terrain eines Kirchhofes Arsenichtsäure enthält, diese Substanz der Leiche sich mittleilen kann oder nicht.

4) Un zu bestimmen, ob die Einführung von Arsenichtsäure in eine Leiche Imbibition des Giftes bewirken kann, die dieselben Erscheinungen hervorbringt, wie die durch Vergiftung.

Wir werden über diese interessanten Fragen uns weiter auf das Protocoll der Commission beziehen.

Dritte Sitzung, am 1. November.

Die Sitzung wurde um 2 Uhr eröffnet. Hr. Orfila zeigte an, dafs er am 28. October $10 \mathrm{Uhr}$ Morgens, vor den Herren Husson, Amusat, Olivier (d'Angers), Soubeiran und Caventou, Mitglieder der Commission, einen Versuch angestellt habe, in der Absicht zu beweisen, dafs Hunde, die durch 10 Centigrm. Arsenichtsänre oder Brechweinstein, als Pulver auf den Schenkel eingerieben, in Zeit von 20-40 Stunden sterben, wenn sie sich selbst überlassen bleiben und keinen Harn lassen; dafs sie aber genesen, wenn es gelingt, dafs sie in Folge wässriger oder diuretischer Getränke viel Urin lassen.

Am 29. Morgens $10 \mathrm{Uhr}$ war einer der mit Arsenichtsäure vergifteten Hunde, der kein Getränk bekommen, und nicht einziges Mal urinirt hatte, gestorben. Der andere, der Wirkung der Arsenichtsäure unterworfene Hund wurde mit diuretischen Mitteln behandelt und schien nicht merklich afficirt zu sein. Einer der mit Brechweinstein vergifteten Hunde war in einem verzweifelten Zustande, obgleich man ihm zu drei verschiedenen Malen diuretische Getränle beigebracht hatte, aber er hatte nicht einziges Mal urinirt. Der andere mit Brechweinstein vergiftete $H$ und befand sich ziemlich wohl; er hatte zweimal in der Nacht urinirt, oline Mithülfe irgend eines Getränkes, und fuhr fort ziemlich häufig zu uriniren; man liels ihn Wasser trinken um die Secretion zu begünstigen. 
Denselben Tag legte man den Oesophagus der beiden kleinen Hunde blofs und unterband ihn, die Ligatur wurde bis zum 30. Morgens $10 \mathrm{Uhr}$ unterhalten. Der mit Brechweinstein vergiftete Hund, der gestern sehr krank erschien, und nicht urinirt hatte, starb in der Nacht vom 29. auf den 30. Die beiden andern Hunde waren fast wieder hergestellt.

Bei Eröffnung der Sitzung zeigte Orfila die vier geheilten Funde, nämlich die beiden, deren Oesophagus am 29. unterbunden war, und die beiden andern, die am 28. durch 10 Centigrm. Arsenichtsäure und Brechweinstein vergiftet waren. Man wird morgen die Leber und den Harn dieser beiden Thiere untersuchen.

Um 10 Uhr weniger 20 Minuten gab man einem ziemlich starken Hunde mittler Grölse 28 Centigrm. Arsenichtsäure, in $130 \mathrm{Grm}$. Wasser gelöst, und unterband den Oesophagus. Nach zwei Stunden war das Thier todt.

Um 11 Uhr weniger 12 Minuten liefs man dieselbe Menge des Giftes, ebenfalls in $130 \mathrm{Grm}$. Wasser gelöst, zwei kleinen Hunden geben, welchen man das Vermögen des Erbrechens gelassen hatte; diesen Thieren wird reines Wasser gegeben werden und sie werden genesen, wenn sie einige Minuten nach der Ingestion des Giftes erbrechen.

Man gab zwei kleinen Hunden 50 Centigrm. feingepulverter Arsenichtsäure, und gab ihnen reines Wasser. Diese Hunde werden drei Stunden nach dem Beginn des Versuchs zur Ader gelassen werden, und genesen, wenn sie gebrochen haben, Einer dieser Hunde wurde um 11 Uhr 4 Minuten und der andere um 11 Uhr $35 \mathrm{Mi}$ nuten vergiftet. Einem andern kleinen Hunde gab man um 11 Uhr 2 Minuten $1 \mathrm{Grm}$. desselben Giftes in festem Zustande. Das Thier, dem man das Vermögen des Erbrechens liefs, wird nur warmes Wasser erhalten, und genesen, wenn es genug erbrechen wird.

Endlich injicirt man in den Magen eines Hundes mîtler Grölse 128 Grm. Fleischbrühe, 64 Grm. Wein 
und eben so viel Branntwein. Diese Injection wird nm $1 \frac{1}{2}$ und $4 \frac{1}{4}$ Uhr wiederholt werden. Das Thier wird innerhalb 24 Stunden sterben, obgleich es brechen kann.

Man schreitet jetzt zur Austrocknung einer faulen in Stücken zerschnittenen menschlichen Leber. Das truckne Product wird in einer Porcellanschale durch $800 \mathrm{Grm}$. concentr. Salpetersäure verkohlt. Die Verkohlung schreitet langsam und schwierig vor, weil die Leber zum Theil schon in Fett verwandelt ist; man erhält aber endlich eine ziemlich trockne Kohle, die man 24 Minuten mit dest. Wasser kochen läfst, worauf das schwärzlich gefärbte Filtrat in einem zuvor geprüften Marsh'schen Apparate untersucht wird, es gab keine Spur Arsenil. Man erhielt nur 5-6 weifse opake Flecken, welche keinen Charainter der Arsenikflecken erkennen liefsen. Nach 25 Minuten des Versuchs gab man in den Apparat einen Tropfen einer Auflüsung von concentr. Arsenichtsäure und fast in demselben Augenblicke setzten sich glänzende Arsenikflecken ab.

Um sich zu überzeugen, ob das reine Kali, dessen man sich bei gerichtlichen Versuchen bedient, Arsenik enthält, liefs man $60 \mathrm{Grm}$. in dest. Wasser auflösen, die Flüssigkeit durch reine Schwefelsäure sättigen und das schwefelsaure Kali in den zuvor geprüften Apparat bringen; es wurde kein Arsenikfleck erhalten, selbst nicht nach Verlauf einer halben Stunde.

Wenn es sich zutragen sollte, bemerkte Hr. Orfila, dafs ein mit Arsenichtsäure vergiftetes Individuum mit Brechweinstein behandelt und gestorben wäre, so könnte es sich in Folge der gerichtlichen Untersuchungen, die mit der Leiche angestellt würden, ereignen, dafs man zugleich Arsenik- und Anlimonialflecken erhielte. Es ist mithin nothwendig, auf diesen Punct unsere Aufmerk samkeit zu richten. Die Arsenik - Antimonialflecken haben ein nach den Verhältnissen des Antimons und Arseniks verschiedenes Ansehn. Beim Vorherrschen von Arsenik sind sie in gewissen Puncten hellbraun, in andern bläulich; beim Vorherrschen von Antimon sind sie dunkel- 
blau, obgleich man dabei auch noch den braunen Stich des Arseniks bemerken kann. Beide, wenn sie ziemlich dick sind, haben eine sehr dunkle Farbe und sind wenig glänzend. Man unterwirft einige dieser Flecken der Einwirkung der Vasserstoffflamme, das Arsenik verflüchtigt sich sogleich, und der Fleck, nun ein einfacher Antimonfleck, zeigt dann die blaue odor graue Farbe cines solchen; er dehnt sich durch die Wirliung der Flamme aus und verhält sich genau wie ein reiner Antimonfleck. Das beste Mittel aber solche gemischte Flecken zu erkennen, besteht darin, sie in reiner und concentr. Salpetersäure aufzulösen, die Auflösung bis zur Trockne zu verdunsten und den gelben Rüclsstand mit liochendem Wasser zu behandeln, welches die Arsenichtsäure in einigen Minaten auflöst, und gelbliche Antimonsäure zurückläfst, die man sich absetzen läfst, worauf man die Auflösung der Arsenichtsäure bis zur Trockne abraucht, und so einen Riickstand erhialt, der mit salpetersaurem Silber sogleich einen ziegelrothen Niederschlag giebt. Die Antimonichtsäure erkennt man sogleich, weun man den gelben Rüclistand mit einigen Tropfen Chlorwasserstoffsäure heifs auflöst, die Auflösung mit etwas Wasser verdünnt und einige Blasen $S c h w$ efelwasserstoffgas einströmen läfst, um sogleich einen orangefarbenen Niederschlag von Schwefelantimon zu erhalten. Diese in der Sitzung angestellte Operation gab genügende Resultate.

Die Schwefelflecken sind gelb, zart und unlöslich in kalter Salpetersïure. Die Eisenflecken sind braun, zart oder glänzend, und lösen sich augenblicklich in Chlorwasserstoffsäure, womit sie ein gelbes Chlorür bilden, welches zur Trockne abgeraucht durch Kalinmeisencyanür blau wird und alle übrigen Charaktere des Eisens darstellt.

Da man angegeben hat, dafs in dem Eisenoxydhydrate der Apotheken wohl Arsenik gefunden sei, wahrscheinlich als Arseniat, und dieses Metall auch in mehren Colcathars existire, so wie in gewissen Eisenvitriolen 
des Handels und auch in einigem kohlensauren Eisen aus Apotheken, so liefs Orfil a anderthalb Stunden lang 4 Unz. arsenikalischen Colcathar (trocknes Eisenoxyd) mit einer Schwefelsäure, die mit $\frac{1}{3}$ ihres Gewichts Wasser verdünnt war, kochen, unter Ersetzen des Verdampften, und die filtrirte Flüssigkeit in einen zuvor geprüften Marsh'schen Apparat bringen; sogleich setzten sich anf der Porcellanscheibe grofse und glänzende Arsenikflecken ab unter einer starken Flamme. Die Flecken waren stets eisenhaltig.

Dieselbe Menge Colcathar, mit dest. Wasser zuvor ausgekocht oder mit Wasser, dem 2 Grm. reines Kali zugesetzt worden waren, gab jetzt in Marsh'schen Apparate keine Spur von Arsenik zu erkennen. "Wie sehr, bemerkte Hr. Orfila, ich auch wünsche, das Verhalten des Eisens, unter dem Gesichtspuncte der gerichtlichen Medicin näher zu entwickeln, so mufs ich doch davon abstehen, weil man dem Hrn. Lafarge Lisenperoxyd und kohlensaures Eisen gereicht hatte, und über den Procels von T'ulle ich mich nicht auslassen mag, als nach dem Beschlufs des Cassationshofes."

In Bezug auf gewisse Todtenhöfe, setzt Hr. Orfila fest:

1) Dafs in einigen dieser Terrains sehr kleine Mengen Arsenik enthalten sind.

2) Dals dieser Arsenik die Bodenmasse nicht verlassen kann, um sich zu den Organen eines darin begrabenen Leichnams zu begeben, weil er sich in der Erdmasse in Form eines in Wasser unlöslichen Salzes findet, und zn seiner Darlegung die längere Einwirkung eines energischen Agens, kochende concentr. Schwefelsäure, nöthig ist und über dem die Haut von giftigen Anflösungen nur chwierig durchdrangen wird, selbst wenn Leichen in Bäder solcher Auflüsungen getaucht werden.

3) Es kann sich ereignen, dafs ein Theil oder auch die ganze Menge Arsenichtsänre, die in der Leiche einer vergifteten Person sich findet, mit der Zeit aus derselben sich entfernt und der Bodenmasse zugeführt wird, weil Arch. d. Pharm. II. Reihe. XXVIII. Bds. 1. Hft. 
das während der Fäulnifs entwickelte Ammoniak die Arsenichtsäure in leichtlösliches arsenichtsaures Salz umändert, welches mit den Flüssigkeiten fortgeführt wird. Es ist leicht begreiflich, dafs dieses Arsenik durch den in dem Boden enthaltenen schwefelsauren Kalk leicht zersetzt und in unlöslichen arsenichtsauren Kalk verwandelt wird.

Nach mehren Erwägungen zur Stütze dieser Behauptungen, liefs Hr. Orfila in einen zuvor geprüften Marsh'schen Apparat eine Auflösung bringen, die durch achtstündiges Kochen von 7 Pfd. der Erdmasse des Kirchhofs Montparnasse mit dest. Vasser erhalten worden war. Die Flüssigkeit gab keine Spur von Arsenik. Eine andere Flüssiglkeit, die dadurch erhalten war, dafs man 24 Stunden lang reine concentr. Schwefelsäure kalt mit der Erdmasse in Berührung und darauf noch 6 Stunden lang damit hatte kochen lassen, und jetzt in einen zuvor geprüften andern Apparat brachte, gab sogleich mehre glänzende Arsenikflecken. Endlich wurde zum Schlurs dieses Gegenstandes gezeigt, dafs das arsenichtsaure Ammoniak den schwefelsauren Kalk sogleich zersetzt, indem sich ein weifser Niederschlag von arsenichtsaurem Kalk bildet.

In Bezug auf die Frage der Imbibition der Leiche, setzte Hr. Orfil a fest, daf's es hinreiche, in den Magen oder das Rectum einer schon erkalteten Leiche eine giftige Flïssigkeit zu bringen, damit diese die Gewebe des Darmkanals durchdringe, und sich dann allmälig in den zunächstliegenden Stellen, nach und nach zu den entfernteren und endlich zu den entlegensten Organen zu verbreiten.

Die gerichtlichen Folgen ans diesen Thatsachen, sagt Hr. Orfila, sind von der gröfsesten Wichtigkeit. Es ist augenfällig, dafs man bei einer Untersuchung in Folge einerVergiftung, melr Gift aus gewissen Organen erhalten wird, wenn die Analyse lange Zeit nach dem Tode unternommen worden ist, als wenn sie 24 oder 36 Stunden nach demselben angestellt wurde, weil im ersten Falle 
die in Rede stehenden Organe, unabhängig von dem während des Lebens erhaltenem Gifte, auch das enthalten, was ihnen durch die Wirkung der Imbibition zugeführt worden ist.

Aber eine anscheinend noch ernstere Sache ist diese. Könnte nicht ein Bösewicht, welcher die Absicht hätte, irgend ein Individuum derVergiftung eines andern anzuklagen, in den Magen und besonders in das Rectum einer Leiche eine giftige Substanz einbringen, welche durch die Analyse gefunden und auf den ersten Anblick es wahrscheinlich machen würde, dafs diese Leiche die einer wirklich vergifteten Person sei? Dieser Fall, zur Ehre der Menschheit sei es gesagt, ist nie vorgelommen. Wenn er aber, gegen jedes Lrwarten, später Gegenstand gerichtlich medicinischer Versuche werden sollte, so würde es doch nicht schwer sein, die Wahrheit in ihr ganzes Licht z.u setzen. Hr. Orfila trug zur Auflösung des Problems eine grofse Zahl Beobachtungen vor, gefolgert aus den Symptomen der Kranken, aus den nach dem Tode constatirten Verletzungen des Zellgewebes, ans der Menge des festen oder flüssigen Giftes, welche im Verdauungskanal gefunden wird, aus der Stelle, welche das Gift in diesem Kanal einnimmt, aus den Wirkungen, welche Gifte auf todte Gewebe hervorbringen, sei es dafs sie kurze Zeit oder mehre Stunden nach dem Tode applicirt wurden, aus dem Unterschiede in der Wirkung der Gifte auf einen lebenden oder einen todten Körper u.s. w. Er schlofs mit einer Betrachtuno, die allein oft hinreicht die Schwierigkeit zu lösen. Bei einem während des Lebens geschehenen Vermiftungsfalle findet man das absorbirte Gift nicht nur in allen Organen, sondern selbst in jedem Theile eines jeden Organs; so giebt die Leber eines durch Arsenichtsïure vergifteten Menschen Arsenik, man mag den obern oder untern oder innern Theil derselben untersuchen. $\mathrm{Is}$ ist nicht so, wenn der Todesfall noch nicht lange her ist, in dem Falle, wo das Gift durch Imbibition bis zur Leber gelangt wäre, dann wird in der That der Theil derselben, 
worauf der Magen liegt, arsenikhaltig sein, der entferntere aber nicht. Und wenn man auch annimmt, dafs in Folge der fortschreitenden Imbibition alle Theile der Leber schon Arsenik enthielten, so werden doch die Lungen, das Herz und besonders das Gehirn erst lange Zeit nach dem Tode solchen enthalten können. Uebrigens ist es auch bekannt, dafs gewisse Gifte durch organische Flüssigkeiten sich zersetzen, so dafs sie unlöslich und in ihrem Fortgange aufgehalten werden. Solchergestalt finden sich die Wirkungen der Imbibition oft gehemmt. Es ist begreiflich, dafs in solchen Fällen, wo nach dem Tode das Gift in den Magen oder das Rectum injicirt war, die von diesen entferntesten Theile nie das kleinste Theilchen der giftigen Substanz erhalten werden.

Zur Stütze dieser Betrachtungen stellte Hr. Orfila folgenden Versuch an:

Die Leiche einer am 27. Octbr. gestorbenen Fran wurde greöfnet, in dere Magen man am 30. um $10 \mathrm{Uhr}$ Morgens eine Auflösung von 60 Centigrm. Arsenichtsäure, in $140 \mathrm{Grm}$. Wasser gelöst, eingebracht hatte. Der Leichnam war von dem Augenblick der Injection an bis auf den heutigen Tag auf der rechten Seite liegen geblieben. Man nahm den obern Theil der linken Lunge weg, schnitt ein Stück von der Oberfläche der Leber und ein anderes von der Unterfläche ab. Alle drei Stücke wurden in drei neuen Porcellanschalen getrocknet, und mittelst reiner concentr. Salpetersäure verkohlt, alle drei verkohlte Massen eine halbe Stunde lang mit dest. Wasser ausgekocht, und die filtrirten Flüssigkeiten in drei zuvor geprüfte Marsh'sche Apparate gebracht. Von der Lunge, so wie von der Oberfläche der Leber erhielt man nicht die mindeste Spur Arsenik, von der Unterfläche der Leber aber, die unmittelbar mit dem Magen in Berührung gewesen war, wurden einige kleine glänzende Arsenikflecken erhalten.

Die Sitzung wurde um $4 \frac{1}{2}$ Uhr aufgehoben. 


\section{Vierte Sitzung.}

Die Sitzung wurde um 10 Uhr eröfnet.

Das Protolkoll der vorigen Sitzung wurde von Hrn. Orfil a vorgelesen und nach Rechtbefund von den Mitgliedern der Commission unterzeichnet.

Es wurden zuerst die beiden am 28. Oct. vergifleten Hunde, von denen der eine 10 Centigrm. Arsenichtsäure, der andere aber so viel Brechweinstein erhalten hatte, vorgenommen; beide Hunde hatten, wie schon gestern bemerkt, beträchtlich urinirt und waren geheilt. Von dem durch Arsenik vergifteten Hunde hatte man ohngefähr 130 und von dem andern $40 \mathrm{Grm}$. Harn erhalten. Beide Flïssigkeiten wurden in neuen Porcellanschalen verdunstet, nachdem sie zuvor mit 10 Centigrm. reinem Kali versetzt worden waren, und das trockne Product wurde über mälsigem Feuer bis zur kaffeebraunen Farbe geröstet. Das Product des Harns von dem Hunde, der Arsenik erhalten hatte, wurde 10 Minuten lang mit dest. Wasser gekocht; das Product des IIarns von dem Hunde, der Antimon erhalten hatte, wurde eine Viertelstunde lang mit reiner Chlorwasserstoffsäure, die mit $\frac{1}{3}$ Wasser verdünnt war, gekocht. Beide filtrirte Flüssigkeiten wurden in zwei zuvor geprüfte Marsh'sche Apparate gebracht, die eine gab Arsenik-, die andere Antimonflecken.

Der Harn des Hundes, der Arsenik erhalten hatte, und der am 31. Oct., also vier Tage nach dem Eingeben des Giftes, gelassen war, denselben Versuchen unterworfen, gab ebenfalls noch $\Lambda$ rsenik zu erkennen. Der Harn des mit Brechweinstein versehenen Hundes, von 29. Oct., den Tag nach der Vergiftung, auf dieselbe Weise behandelt, gab sogleich Antimon zu erkennen. Die Lebern dieser Thiere, getrocknet und verkohlt durch reine concentr. Salpetersäure, lieferten zwei kohlige Massen, die man 25 Minuten mit dest. Wasser kochen liefs. Die filtrirten Flüssigkeiten wurden in Marsh'sche Apparate gebracht, sie gaben keine Spur von Arsenik und 
Antimon zu erkennen, selbst nachdem der Versuch eine halbe Stunde gedauert hatte.

Ohngefähr $120 \mathrm{Grm}$. Urin von einer Fran, am 1. Nov. gelassen, die wegen einer Hautkrankheit auf Anrath des Dr. Emery seit $2 !$ Monat täglich sehr kleine Dosen der Fowler'schen Auflüsung (eine Zusammensetzung von Kali und Arsenichtsäure) genommen hatte, wurde den erforderlichen Operationen unterworfen. Man erhielt einige Arsenikflecken. Nach dem gleichen Verfahren erhielt man aus 2 Portionen von Hrn. Dr. Bouvier eingesandlen Harn, der von einer an Pneumonie leidenden Frau herrührte, der der Arzt einmal 20 und ein andermal 30 Centirrm. Brechweinstein verordnet hatte, deutliche Antimonflecken.

Der Vorgang bei den Menschen ist also wie bei den Hunden, sagt Hr. Orfila, in beiden Fällen sieht man die durch den Macren eingebrachten arsenikalischen und antimonialischen Materien durch den Urin ausgesondert werden. Wir haben dieses schon in mehren Fallen bei dem Harn von Kranken beobachtet, die von den Herren Dumepil, Hus son und Bouvier behandelt wurden. Ueberdies haben wir beobachtet, dass die Leber zweier Individuen, die nach der Administration zweier starker Gaben von Brechweinstein gestorben waren, merkliche Mengen von Antimon enthielten.

Ich möchle ernstlich die Aufmerksamkeit der Versammlung auf die gerichtlichen Folgerungen, die aus diesen Thatsachen hervorgehen, richten. Die hier vorgenommenen Versuche beweisen unwiderleglich, dafs, wenn wir die beiden am 28. Oct. vergifteten Hunde einige Stunden oder einige Tage nach der Vergiftung anfgehangen hätten, wir aus der Leber wie aus den andern Eingeweiden dieser Thiere Arsenik und Antimon erhalton haben würden. Heute, am sechsten Tage nach der Vergiftung, finden wir kein Atom dieser Metalle mehr in den bemerkten Eingeweiden; aber der seit dem 29. bis auf den hentigen ' $\mathrm{Tag}$ ausgegebene Harn enthält $A \mathrm{r}$ senik und Antimon. Ls kann sich also ereignen, dafs 
ein Individunm durch die eine oder andere dieser Substanzen vergiftet, stirbt, und dafs man keine Spur des Giftes in der Leber, der Milz, dem Herzen u. s. w. findet, weil das Individuum noch mehre Tage lebte und alles absorbirte Gift durch den Harn und vielleicht auch durch andere Wege wieder ausgeführt worden ist. Wie sehr würde man sich also betrïgen, wollte man sagen, daf's die beiden Hunde, auf deren Schenkel am 28. Oct. Arsenichtsäure und Brechweinstein applicirt wurden, nicht vergiftet gewesen wären, blofs, weil man 6 Tage nach der Vergiftung in der Leber, der Milz 11. s. w. die Existenz von Arsenil oder Antimon nicht aufgefunden hätte! Ich will nicht behaupten, dafs stets am sechsten Tage die Organe von dem absorbirten Gifte wieder frei sein wiirden, denn es giebt hier grofse Differenzen, je nach den Constitulionen und der Dosis des Giftes, und nach der Menge des in den ersten Tagen der Vergiftung gelassenen Harns.

Ir. Orfil a zeigte darauf die beiden Hunde, deren Oesophagus am 29. unterbunden und die jetzt vollkommen geheilt waren. Von den fünf, die Tags zuvor mit Arsenichtsäure vergiftet wurden, durch 50 Centigrm. und durch 1 Grm. des gepulverten Giftes, sind vier völlig wieder hergestellt. Der fünfte, welcher schon drei Stunden nach der Vergiftung ziemlich wohl sich befand, starb gestern um I Uhr plötzlich, weil das ihm injicirte warme Wasser statt in den Magen in die Luftröhre lkam, in Folge der Erstickung, wie auch die in Gegenwart der Mitglieder der Commission gemachte Leichenöffnung bewies. Die geheilten vier Hunde mufsten zu wiederholten Malen erbrechen und wurden nur mit warmem Wasser behandelt, ausgenommen zwei, die um 1 $1 \frac{1}{2}$ Uhr zur Ader gelassen wurden; der eine verlor 25, der andere $70 \mathrm{Grm}$. Blut.

Der Hund, welchem man gestern um $10 \frac{1}{2}$ Uhr Morgens das tonisch - excitirende Medicament beigebracht hatte, starb $6 \frac{1}{2}$ Uhr Abends.

Um $10_{s}^{\frac{1}{3}}$ Uhr brachte man in den Magen zweier 
Hunde 25 Centigrm. in Wasser gelöster Arsenichtsäure und unterband den Oesophagus. Um $11 \frac{1}{4}$ Uhr wurde die Ligatur weggenommen und nicht wieder ersetzt, aber ein Gemenge von $29 \mathrm{Grm}$. Fleischbrühe, $32 \mathrm{Grm}$. Branntwein, eben so viel Wein und 15 'Tropfen Laudanum liquidum Sydenh. injicirt. Diese Operation wurde um 1,3 und $5 \frac{1}{2}$ Uhr wiederholt. Das eine dieser Thiere starb um 6 Uhr, obgleich es seit Mittag wirklich gebrochen hatte; das andere starb um $7 \frac{1}{4} \mathrm{Uhr}$, dieses war weit stärker als das andere.

Diese Versuche, sagt Orfila, führen -mich natürlich darauf, über die Behandlung von Vergiftungen durch Arsenikalien zu sprechen.

Nach einigen Betrachtungen über die Virkungsweise der Gifte, sagt Orfila, dafs der zu einem Vergiftungsfalle herbeigerufene Arzt damit anfangen murs, den Kranken oben und unten auszuleeren, um aus dem Verdauungskanal den Theil des Giftes zu entfernen, der noch nicht zur Wirksamkeit gelangt ist; denn wenn man von dieser Portion denselben nicht befreit, so mufs sie eine schädliche Wirkung ausüben, und mit jeder Minute Verzug die Zufälle verschlimmern. Warmes Wasser, Brechweinstein und Purgiermittel sind hier fast immer angezeigt. Gegen gewisse Gifte, die man im Verdauungskanal sogleich zersetzen kann, indem man sie in unlösliche und unwirksame Materien umbildet, gebraucht man mit Vortheil Gegengifte und darauf leichte Brechmitiel and Abführungsmittel, welche zugleich den nicht zersetzten Theil des Giftes wegführen. So reicht es bei Vergiftungen durch Blei oder Baryt hin, einige Grane schwefelsaures Kali oder schwefelsaures Natron nehmen zu lassen, um diese Gifte sogleich in schwefelsaures Blei oder schwefelsauren Baryt zu verwandeln und unschädlich zu machen. In gewissen Fällen nimmt man seine Zuflucht zu solchen Milteln, welche die Eigenschaft haben, die giftige Substanz, zugleich zu neutralisiren und aus dem Verdauungskanal zu entfernen, wie z. B. in Vasser verbreitetes Eiweifs bei Vergiftungen durch 
Quecksilber - und Kupfersalze (Aetzsublimat und Grünspan).

Man mufs aber hierbei nicht stehen bleiben. Ein Theil des eingenommenen Giftes kann schon absorbirt und mit dem Blute zu allen Organen des Körpers geführt worden sein: und das ist der Theil des Giftes, welcher die grölsten Schwierigkeiten und Leiden bewirkt, and zu dessen Entfernung Brechnittel und Abführungsmittel leider unwirksam sind. Iieser Theil des Giftes bewirkt mehr oder weniger bedenkliche Erscheinungen, die man ohne Verzug bekämpfen muls, sei es durch Blutegel und mildernde Mittel, wenn die Krankheit inflammatorisch ist, sei es durch narkotische oder reizende, und, nach meinen Erfahrungen, vorziiglich durch diuretische, in Verbindung mit dem einen oder andern angegebenen Verfahren. Wir wollen einen Augenblick bei der diuretischen Behandlung verweilen.

Seit es erwiesen ist, dafs bei den meisten Vergiftungen das Gift absorbirt und jeder Fiber des Körpers zugeführt wird, wo es eine gewisse Zeit bleibt, und dann, wenn nicht ganz, doch zum gröfsten Theil durch den Urin entfernt wird, so zeigt die einfache gesunde Vernunft an, dafs es angremessen ist, die Harnsecretion z,u befördern, um diesen Theil des Giftes unauflöslich und in kleinen Portionen zu entfernen, welcher gewissermafsen jede Fiber vergiftet und das Individnum tödtet, wenn die Medicin nicht Herr darülser wird. Die zahlreichen Versuche an 'Thieren bestätigen die Richtigkeit dieses Satzes, sic beweisen, dafs alle Hunde, die mit so viel Arsenichtsäure oder Brechweinstein vergiftet waren, dafs sie danach binnen $20-40$ Stunden sterben mufsten, ziemlich schnell genasen, wenn nian sie merklich zum Uriniren bringen konnte, mittelst eines Diureticums ans 5 Liter Wasser, 1 Liter weifsen Vein, einer Flasche Salzwasser und $60-89 \mathrm{Gr}$. salpetersaurem Kali, in Dosen von 1-2 Glas gegeben.

Auf die Behandlung endlich der Vergiftung mit Ar. senichtsäure übergehenú, fragt Orfila, ob wirlilich ein 
Gegengift für diese existire; er verneint solches. Schwefelsïure, Schwefelallralien, Kohle, Kalkwasser u. s. w. sind unwirksam, oft gefährlich. Das Eisenoxydhydrat scheint ihm ebenfalls nicht geeignet, weil man es in hohen Dosen anwenden muls, und es seiner Unlöslichkeit wegen sich schwierig mit der Arsenichtsäure verbindet, ferner, weil es oft selbst arsenikalisch ist, und weil es oft besser ist, schnell Erbrechen und Stuhlgang zu bewirken und die im Verdauungskanal enthaltenen arsenikalischen Partikeln zu entfernen.

Welche Behandlmysweise murs angewendet werden, wenn die Krankheit durch bereits absorbirte Arseuichtsäure entwickelt ist? Es bieten sich uns drei Systeme dar: 1) die antiphlogistische, 2) die tonische und 3) die diuretische Methode.

Die seit den undenklichsten Zeiten angepriesene antiphloyistische Methode hat bei Menschen vielfach guten Erfolg gehabt. Der $\Lambda$ derlal's ist ron unzweifelhaften Nutzen gewesen, in Fällen anģenfälliger Reaction, wenn die Haut warm, der Puls stark und schnell, das Gesicht roth und aufgetrieben, Delirium n. s. w. sich gezeigt hatte. Von der Anwendung dieser Methode habe ich schon 21 Erfolge gehabt. In englischen medicinischen Journalen findet man die Angabe, dafs auf 19 Arsenikvergiftungen, die seit einigen Jahren in medicinische Behandlung kamen, 18 durch Aderlafs geheilt wurden. Endlich steht der Dr. Schedel, welcher so oft die Wirkungen der im Hospital St. Louis so hänfig vorkommenden medicinischen Anwendung des Arseniks zu beobachten Gelegenheit hat, nicht an, über den Aderlafs günstig und gegen die Tonica sich auszusprechen. Und was setzt man so zahlreichen und wohlbewahrbeiteten Thatsachen entgegen? Einige Erfahrungen an Hunden, denen man starke Dosen von Arsenik eingegeben hatte, und die nach Aderlassen starben. Diese Versuche sind aber so schlecht angestellt und so leicht beschrieben, dafs nan keine Riicksicht darauf nehmen kann. Die Thiere, die man so behandelt hatte, hatten keinen 
Tropfen Wasser noch sonst eine Flüssigkeit bekommen, welche das Erbrechen begünstigt hätte. Die Aderlässe sind oft erst kurze Zeit nach der Vergiftung angestellt, wo sie die Absorption begïnstigen mul'sten. Betrachten Sie die Thatsachen, wovon Sie Zeugen waren, sehen Sie, ob die vergifteten und zur Ader gelassenen Thiere nicht geheilt sind; ich will damit nicht behaupten, dafs sie ihre Herstellung dem Aderlass verdankten, weil sie erbrachen, und neues Erbrechen durch warmes Wasser hervorgerufen wurde; aber stets haben die Aderlässe hier die Heilung nicht verhindert, und es ist deshalb thöricht, sie als verderblich oder tödtlich zu verbannen. Im Ganzen erhellet, dafs der Arzt die durch Arsenichtsäure vergifteten Kranken zur Ader lassen und mildernde Mittel geben muls, wenn deutliche Symptome von Reaction sich zeigen.

Ras ori ist der erste, welcher das tonische Verfahren bei Arsenilvvergiftungen, als zur Heilung besonders geeignet, angegeben hat; nach ihm soll dieses Gift asthenisch oder schwächend wirken. Worauf stützt man sich hier? Auf einige Erfahrungen an Hunden. Von 19 mit Arsenichtsäure, in Wasser aufgelöst, vergifteten Hunden wurder 11 durch tonische Mittel geheilt. Ich nehme diese Thatsachen an; aber sie beweisen nichts zu Gunsten des Systems, denn alle genesenen Thiere erbrachen heftig, und wir wissen, dafs sie eben sowohl genesen sein würden, wenn man ihnen warmes Wasser würde gegeben haben, dafs sie zum Erbrechen gekommen wären, and niemand wird das Wasser für ein Excitans halten. Haben wir unsererseits nicht gesehen, dafs zwei durch 25 Centigrm. Arsenichtsäure vergiftete Hunde in einigen Stunden starben, obgleich sie mit tonischen Mitteln behandelt wurden. Zur Verhinderung der Heihng genügte, während der ersten Stunde nach der Vergiftung das Erbrechen durch Unterbinden des Oesophagns nu verhindern. Die tonischen Mittel sind also völlig unmureichend, wenn sie IIunden, dic nicht erbrachen, in der ersten Stunde nach der Vergíftung gereicht werden; 
waren sie in einigen Fällen von Erfolg, so waren die Thiere schon zum Erbrechen gelommen, bevor die tonischen Mittel gereicht wurden, dals sie aufs neue Erbrechen erregten, oder die Harnsecretion beförderten. Der Arzt murs also von dieser Behandlungsweise absehen.

Für die Vorzüge der diuretischen Methode habe ich nur noch wenig zu bemerken. Sie ist unschädlich, rationell, und von Erfolg, wenn sie die Harnsecretion angemessen vermehrt. Jedoch sie ist bei Menschen noch nicht versucht worden. Alles aber lälist erwarten, daf's sie bei Menschen denselben Erfolg haben werde, als bei Hunden, weil auch bei den Menschen die Organe aljsorbirte $\Lambda$ rsenichtsïure wieder abgeben, die dann durch den Harn eliminirt wird.

Die Sitzungs warde um $4 \frac{1}{2}$ Uhr geschlossen. *)

\section{Die Aufsuchung des Arsens in den zweiten Wegen ; \\ von}

Dr. Meurer in Dresden.

Die durch die Fortschritte in der Wissenschaft herbeigefuihrte Nothwendigkeit, den Arsen oder auch andere anorganische Gifte, wemn man dieselben bei gerichtlichen Untersuchnngen, nicht mehr in den ersten Wegen, d. h. im Schlund, Magon und Darmkanal aufzufinden vermag, diese Stoffe in den zweiten Wegen aufzusuchen, veranlafste mich, da mir zuw eilen gerichtliche Arbeiten dieser Art übertragen werden, zu den hier zu beschreibenden Untersuchungen.

Ich hatte hierbei den doppelten $Z Z_{W}$ eck, crstens nämlich diejenigen Organe oder Flüssiggkilen genaner liennen zu lernen, wo man den Giftstorf vorzugswcise aufzusuchen habe, und dann die verschiedenen Verfahrungessarten der Zerstörung der organischen Stofe und die Arten den Arsen darzuthun, zu prüfen, um bei vor-

*) Journ, de Chim, mod. 2. Ser. VI, 690. 